\title{
Múltiples Perspectivas Sobre un Problema Complejo: Comentarios Sobre Cinco Investigaciones en Violencia Escolar
}

\author{
Multiple Perspectives on a Complex Problem: \\ Comments on Five Studies on School Violence
}

\author{
Enrique Chaux \\ Universidad de los Andes, Colombia
}

\section{El Problema de la Violencia Escolar Requiere Múltiples Perspectivas}

La violencia en el contexto escolar es un problema serio en toda América Latina. Varios estudios han mostrado prevalencias altas en países como Argentina, Colombia, Chile y México (e.g., Aguilera, Muñoz \& Orozco, 2007; Chaux \& Velásquez, 2008; Kornblit, Adaszko \& Di Leo, 2008; Madriaza, 2008). Hoy sabemos, también, que la agresión que sufren muchos estudiantes puede tener consecuencias académicas y psicológicas graves en el corto y largo plazo (e.g., DeLuca, Pigott \& Rosenbaum, 2002, Abril; Forero, McLellan, Rissel \& Bauman, 1999; Gladstone, Parker \& Malhi, 2006). Somos conscientes de que no es fácil lograr disminuciones efectivas en la agresión. Incluso, algunas evaluaciones rigurosas no han encontrado cambios significativos en programas bien estructurados (Social and Character Development Research Consortium, 2010). Es decir, es un problema serio y muy prevalente, que debemos comprender mejor para identificar, ensayar y evaluar constantemente nuevas alternativas para prevenirlo. Este compendio es una muestra clara del tipo de investigaciones que necesitamos para avanzar hacia esa comprensión.

Una de las principales contribuciones de este compendio de artículos es la diversidad de perspectivas que presenta. Un problema tan complejo como la violencia escolar requiere una mirada integral que abarque las perspectivas de los distintos actores involucrados. Varios artículos de este volumen incluyen la perspectiva de dos de los actores centrales de las dinámicas escolares: los estudiantes (Dias, Lisboa, Koller \& DeSouza; López et al.; Potocnjak, Berger \& Tomicic) y los profesores (López et al.; Pérez). Adicionalmente, otros actores, como directivas, orientadores, psicólogos, asistentes de la educación, padres, madres y apoderados, fueron incluidos en la investigación de López et al. Esta diversidad de miradas sobre el problema genera una gran riqueza de información que no es común en trabajos académicos sobre el tema, los cuales tienden con frecuencia a estudiar exclusivamente a los estudiantes.

Otra gran fortaleza de este grupo de investigaciones es la diversidad de metodologías empleadas. Entre los métodos de recolección de datos usados están cuestionarios basados en nominaciones (Dias et al.), cuestionarios de autoreporte de conductas (Varela), cuestionarios de reporte de observación de conductas entre compañeros (Varela), entrevistas individuales semi-estructuradas (López et al; Potocnjak et al.), grupos focales (López et al; Potocnjak et al.), encuestas basadas en viñetas (Pérez), observaciones no participantes (López et al.) y revisión de documentos (López et al.). Esto permite una diversidad de tipos de análisis que van desde estrategias cuantitativas, como las ecuaciones estructurales (Dias et al.), hasta estrategias cualitativas, como la teorización enraizada (Potocnjak et al.) o la producción de repertorios

Enrique Chaux, Departamento de Psicología, Universidad de los Andes, Bogotá, Colombia.

La correspondencia relativa a este artículo debe ser dirigida a Enrique Chaux, Departamento de Psicología, Universidad de los Andes, Carrera 1 Nº 18A-12, Bogotá, Colombia. E-mail: echaux@uniandes.edu.co 
interpretativos (López et al.). Todo esto representa un gran avance en un campo dominado principalmente por los análisis cuantitativos basados en datos recogidos exclusivamente por cuestionarios.

A continuación busco destacar algunos de los aportes que hace esta serie de artículos a la comprensión del fenómeno de la violencia escolar, particularmente desde la perspectiva de los estudiantes, los profesores, la comunidad educativa y el contexto cultural. En cada caso, además de señalar los avances más relevantes, busco identificar implicaciones prácticas para el diseño de intervenciones, así como posibles vacíos que podrían ser cubiertos por futuras investigaciones. Finalmente, presento algunas sugerencias para políticas públicas que podrían derivarse de los resultados encontrados.

\section{Perspectiva de los Estudiantes}

Como una adolescente lo señala en el estudio de Potocnjak et al., "ustedes los grandes no saben lo que pensamos nosotros, no nos toman en cuenta". Esta queja es válida y resulta irónico pretender promover la convivencia pacífica entre los estudiantes sin escucharlos atentamente. Algunos de los estudios de este volumen contribuyen a superar esta limitación.

Varios de los estudiantes entrevistados muestran que la violencia escolar no solamente involucra a unos pocos estudiantes agresivos, sino que implica una dinámica grupal. Ellos describen el papel fundamental que juegan diversos roles, como la "fianza" (defensores), los "carboneros" (instigadores) y los testigos (Potocnjak et al.). Estos roles son similares a los definidos por Christina Salmivalli y su grupo en Finlandia (Salmivalli, Lagerspetz, Björkqvist, Österman \& Kaukiainen, 1996) con respecto a situaciones de bullying: a) víctimas: sufren la intimidación; b) intimidadores líderes: inician y lideran la intimidación; c) asistentes: ayudan a el/la líder de la intimidación, por ejemplo, reteniendo a la víctima o participando en la intimidación después de que el/la líder la ha iniciado; d) reforzadores: al reírse, al incitar o, simplemente, al servir de audiencia, terminan reforzando la intimidación; e) defensores: intervienen para frenar la intimidación, para defender de maneras no agresivas a la víctima o para consolar a la víctima una vez la agresión ha terminado y f) externos: se alejan de las situaciones de intimidación o no están presentes cuando ocurren. Tal como lo han mostrado varios estudios, las dinámicas de agresión dependen sustancialmente de lo que hagan esas otras personas que están alrededor (Craig \& Pepler, 1997; Hawkins, Pepler \& Craig, 2001). Esto tiene implicaciones muy relevantes para las intervenciones porque, en vez de buscar directamente que los agresores dejen de serlo, puede ser más efectivo lograr que algunos observadores pasivos decidan asumir el rol de defensores y, al hacerlo, frenen una dinámica que reforzaba la agresión. El hecho de que algunos adolescentes en el estudio de Potocnjak et al. indiquen que los roles son móviles da más impulso a esta alternativa. Además, algunos programas de prevención basados en esta idea, como KiVa (Kärnä et al., 2011; Salmivalli, Kärnä \& Poskiparta, 2009) o Aulas en Paz (Chaux, en prensa), han mostrado resultados importantes.

Algunos adolescentes entrevistados indicaron que la agresión puede brindar popularidad y estatus social, es decir, a veces puede ser funcional y adaptativa (Potocnjak et al.). A pesar de que Dias et al. no encontraron una relación significativa entre agresión y popularidad, varios estudios han mostrado que algunos adolescentes pueden ser simultáneamente populares y agresivos, mientras otros pueden ser populares y prosociales (e.g., Rodkin, Farmer, Pearl \& Van Acker, 2000). El hecho de que la agresión pueda ser funcional al traer beneficios en sus grupos no quiere decir, sin embargo, que deba ser aceptada. Entender qué es lo funcional en la agresión es central, porque ayuda a comprender mejor sus orígenes y saber mejor cómo prevenirla. La agresión debe prevenirse siempre porque puede generar daños físicos, emocionales y/o sociales en quienes la reciben. Intervenciones que promuevan que los estudiantes no valoren la agresión de sus compañeros, sino que, en cambio, promuevan la popularidad de quienes actúen de manera prosocial, podrían ser muy efectivas porque llegan directamente a aquello que más buscan muchos niños, niñas y adolescentes: el reconocimiento de sus pares. 
Varios estudiantes entrevistados resaltaron la importancia del rol que pueden cumplir los demás actores de la comunidad educativa, especialmente profesores y padres. Sobre los padres y madres de familia, por ejemplo, destacaron la necesidad de que puedan brindar afecto, apoyo, confianza y atención (Potocnjak et al.). Con respecto a los profesores, mencionaron que deberían ser fuente de autoridad y afecto, simultáneamente. Estas ideas son muy coherentes con estudios que indican que los estilos parentales y docentes que combinan autoridad con afecto están asociados con un mejor desarrollo emocional, social y académico (Baumrind, 1973; Hughes, 2002). Estos son estilos en los que sí hay límites y normas claras que se aplican consistentemente, pero estos límites y normas son concertados con los niños o adolescentes. Además, en estos estilos las relaciones padres-hijos o profesores-estudiantes se caracterizan por el afecto y la comunicación, nunca por el maltrato o la imposición por la fuerza. Esto indica que las intervenciones deben llegar tanto a padres como a profesores, buscando promover este tipo de estilos parentales y docentes, dado que muchos padres o profesores creen erróneamente que solo hay dos extremos: o ser autoritario, imponiendo el orden, a veces incluso por la fuerza, o ser permisivo, brindando cariño y dando libertad de acción. En cualquier caso, los comentarios de los estudiantes resaltan la importancia de que las intervenciones sean integrales, buscando involucrar al resto de la comunidad educativa, especialmente a los padres y profesores. Este tipo de intervenciones integrales son cruciales, como lo muestra Varela en su evaluación.

Tomar en cuenta la perspectiva de los estudiantes es fundamental. Sin embargo, sería un error implementar directamente sus recomendaciones sin pasar por un proceso de análisis crítico y evaluación. Por ejemplo, como lo muestran Potocnjak et al., algunos adolescentes parecen creer que la agresión es la mejor manera para ejercer la disciplina y frenar la agresión, que los padres tienen derecho al maltrato ("ellos tienen más derecho"), o que a veces las víctimas se merecen la agresión que reciben ("algo habrá hecho"). Es claro que si no se toma en cuenta la visión de los estudiantes se pueden generar visiones sesgadas y limitadas sobre la realidad, pero también es claro que no todas las sugerencias de los estudiantes son coherentes con lo encontrado en estudios rigurosos. Quizás se requiere escucharlos, pero se deben tomar sus perspectivas y recomendaciones como hipótesis que siempre deben verificarse con nuevos estudios y evaluaciones que involucren, idealmente, diversas perspectivas y enfoques metodológicos.

\section{Rol de los Profesores}

Los profesores están en un lugar privilegiado desde el cual pueden contribuir a prevenir la agresión y promover la convivencia, como algunos lo reconocen (López et al.). Es claro que este lugar privilegiado no puede significar que deban asumir todo el peso de la responsabilidad. La prevención de la violencia debe ser una responsabilidad compartida entre la familia, la escuela y la sociedad en general y se necesita que cada uno cumpla su parte para que se puedan realmente lograr cambios de manera integral. Sin embargo, López et al. reportan que algunos profesores parecen responsabilizar de los problemas de violencia escolar a factores externos incontrolables, como la existencia de casos de estudiantes problemáticos, padres despreocupados, instalaciones físicas insuficientes o, incluso, un sistema educativo deficiente (López et al.). Esto puede llevar a que algunos profesores se desliguen de su responsabilidad, al creer que no van a poder contribuir a mejorar la convivencia. En otras palabras, si tienen una baja autoeficacia para generar cambios (es decir, si no creen en su propia capacidad para lograrlo; Bandura, 1989), pueden llegar a considerar que esa no es su responsabilidad. Por ejemplo, quienes creen que la violencia escolar se debe solamente a estudiantes problemáticos que tienen padres despreocupados con frecuencia consideran que la solución es sacar a esos estudiantes de la escuela, aunque, al hacerlo, estarían renunciando a su función formadora y trasladando el problema a otro colegio o a la calle.

Visiones más complejas sobre el problema de la violencia escolar podrían, en cambio, brindar más alternativas a los profesores sobre el rol que podrían asumir en la promoción de la convivencia. Por ejemplo, cuando la agresión escolar se ve como una dinámica grupal, la solución preferida no es sacar a algunos, sino cambiar lo que el grupo valora o cómo el grupo responde 
ante las situaciones de agresión. Una formación de profesores que promueva la comprensión de que un estudiante recurre con frecuencia al bullying como una manera para conseguir reconocimiento y popularidad en su grupo puede ayudar a que consideren nuevas alternativas como, por ejemplo, generar acuerdos con toda la clase para que los observadores rechacen cualquier acto de agresión de sus compañeros, en vez de derivar al departamento de psicología, orientación o a instancias disciplinarias a los agresores o a las víctimas. Este tipo de alternativas ayuda a recuperar la creencia de los profesores en que ellos sí pueden cumplir un rol central en la promoción de la convivencia desde sus salas y escuelas.

Los estudios presentados en esta compilación también parecen indicar que muchos profesores necesitan una mejor comprensión sobre los tipos de agresión y sus dinámicas. Pérez muestra que, aunque la mayoría comprende la gravedad de las situaciones de bullying físico y verbal, algunos no le dan la misma importancia al bullying relacional y están menos dispuestos a intervenir en esos casos. Algunos profesores hombres, por ejemplo, parecen empatizar más con víctimas de bullying físico que con víctimas de bullying verbal o relacional, mientras que profesoras mujeres empatizan por igual con las víctimas de todos los tipos de bullying. Esto es muy relevante porque los estudiantes requieren que los profesores, quienes en muchas situaciones representan para ellos la única figura de autoridad accesible, les brinden todo el apoyo ante cualquier situación de bullying, incluso cuando no tienen heridas físicas que mostrar. Los procesos de formación de profesores deberían prepararlos para que puedan identificar los distintos tipos de agresión, comprender las graves consecuencias que todo tipo de bullying puede generar y estar dispuestos a intervenir para que ningún tipo de maltrato ocurra entre sus estudiantes. Asimismo, es importante que la formación de docentes les ayude a diferenciar el bullying de situaciones de agresión que no son repetidas o sistemáticas y a diferenciar el bullying de conflictos, dado que las respuestas útiles para el manejo de conflictos, como, por ejemplo, la mediación por parte de pares, no son recomendables para el manejo del bullying (Chaux, en prensa).

Todo lo anterior resalta la importancia del rol que tienen los profesores en la promoción de la convivencia escolar. Los profesores de todas las áreas deben enfrentar con mucha frecuencia situaciones de agresión y maltrato en sus clases y la manera como respondan es crucial para la dinámica que tomarán estas situaciones. Un profesor que no responda ante situaciones de agresión en su clase o que, incluso, participe en alguna burla, ofensa o maltrato, estará, como mínimo, desperdiciando una oportunidad importante de promocionar la convivencia pacífica y de prevenir futuras situaciones de agresión y violencia. Sin embargo, es poca la formación que reciben los profesores sobre cómo responder constructivamente ante este tipo de situaciones. De hecho, la queja principal que tienen muchos profesores sobre la formación que recibieron en facultades de educación es que no les enseñaron estrategias para manejar asuntos de agresión y disciplina en sus clases (Jones, 2006). En un futuro cercano todos los currículos de formación de profesores deberían incluir cursos y prácticas específicas sobre este tema, si realmente queremos que todos tengan la capacidad para asumir de manera responsable y efectiva el rol que tienen.

\section{La Comunidad Educativa}

La violencia escolar requiere ser trabajada de manera integral, incluyendo a los estudiantes, profesores, orientadores y otros profesionales, directivas y familias. No es una responsabilidad exclusiva de los orientadores, como parecen sugerirlo varios de los participantes en el estudio de López et al., especialmente si tomamos en cuenta que las dinámicas de grupo que sustentan la mayoría de las situaciones de agresión no se pueden frenar con intervenciones individuales. Eso hace particularmente difícil la solución cuando no se logra una coordinación de esfuerzos desde los distintos frentes. De hecho, es común que, en vez de coordinación, haya acusaciones mutuas del estilo: "esto es responsabilidad de los padres, ellos nos los traen mal educados" o "esto es responsabilidad del colegio, para eso lo llevo allá, para que lo eduquen". Justamente por esta razón, las intervenciones deben involucrar a todos los actores, como la 
descrita por Varela o las recomendadas consistentemente por Olweus (1993). Intervenciones que han mostrado impactos positivos en el largo plazo, como el programa de prevención de Montreal, actúan de manera simultánea en la escuela y en las familias, incluso realizando visitas frecuentes a los hogares de las familias de los niños que muestran mayores problemas de agresión (Chaux, 2005; Vitaro, Brendgen \& Tremblay, 2001).

Asimismo, las intervenciones integrales no solamente deben buscar mejorar el clima en las aulas, sino también el clima escolar en general. Por ejemplo, si se quiere promover que los estudiantes sean capaces de manejar sus conflictos de manera pacífica, los adultos de la institución también lo deben hacer, no solamente en los conflictos que tienen con sus estudiantes, sino también en los conflictos entre ellos: entre profesores, entre profesores y directores, entre directores y padres de familia, entre otros. Es decir, las intervenciones deben crear mecanismos de promoción de un clima escolar pacífico incluyendo, por ejemplo, capacitación en el manejo de conflictos para directivos, profesores y demás adultos de la comunidad educativa. De esta manera, se estaría enviando un mensaje de consistencia que, no solamente ayuda al desempeño laboral, sino a la formación para la convivencia pacífica de todos. Y este mensaje debería empezar desde los primeros años, dada la enorme importancia que tiene la prevención temprana de la agresión (Tremblay, Gervais \& Petitclerc, 2008).

\section{El Contexto Cultural}

Los estudios presentados en este compendio son todos del contexto chileno, excepto el de Dias et al., realizado en Brasil. En términos estrictos, no es posible generalizar los resultados encontrados a otros contextos, ni siquiera a todo Chile, que en sí tiene una gran diversidad de contextos culturales y escolares. Sin embargo, hay muchas similitudes entre lo encontrado en estos cinco estudios y lo reportado por gran parte del campo de estudio sobre agresión escolar. Pareciera haber más similitudes que diferencias, lo cual podría sugerir que las dinámicas detrás del fenómeno de violencia escolar pueden ser más comunes de lo que podríamos pensar dadas las diferencias culturales.

A pesar de las similitudes, no es posible asumir los resultados de estudios realizados en un contexto y usarlos en otros, sin antes haber realizado una verificación de su aplicabilidad. Esto resalta la importancia de estudios como el de Pérez que buscaba replicar y ampliar lo encontrado por otros estudios internacionales sobre la menor relevancia que algunos profesores le asignan al bullying relacional. Luego de esta réplica, se puede reafirmar con mayor firmeza la importancia de realizar procesos de formación de profesores para promover su comprensión del efecto negativo que puede generar el bullying relacional.

El estudio de Dias et al. no logró replicar resultados de otros estudios sobre la relación directa entre popularidad y agresión o sobre la prosociabilidad como factor protector contra el maltrato. Esto puede indicar que en el contexto cultural brasilero donde fue realizado el estudio no se valora la agresión de la misma manera que en otros contextos. De hecho, en su estudio original, Rodkin et al. (2000) encontraron diferencias en la relación entre popularidad y agresión en diversos contextos culturales. Sin embargo, también es posible que la falta de relación entre popularidad y agresión se deba a diferencias metodológicas con otros estudios. Por ejemplo, Dias et al. usaron técnicas estadísticas que buscan asociaciones entre variables (ecuaciones estructurales), mientras que Rodkin et al. (2000) usaron técnicas que agrupan personas con características similares (análisis de conglomerados o clusters). Nuevos estudios comparando contextos distintos, pero usando la misma metodología, se requieren para aclarar mejor la relación entre popularidad y agresión o para identificar mejor el rol de la prosociabilidad como posible factor protector.

Asimismo, Varela realizó una evaluación de un modelo de intervención desarrollado en Estados Unidos, pero traducido y adaptado al contexto chileno. Realizar este tipo de evaluaciones es fundamental, así las intervenciones hayan sido muy exitosas en otro contexto. Sin evaluaciones no sabemos si las acciones están teniendo el efecto buscado. Recientemente, Farrington y Ttofi (2009) llevaron a cabo un meta-análisis de programas contra el bullying y encontraron 
que los programas inspirados en el trabajo de Dan Olweus que han sido tan exitosos en Noruega y en otros países europeos, no lo están siendo en Estados Unidos. Todavía no es claro qué genera estas diferencias, pero una de las posibles explicaciones es que los profesores en Estados Unidos tienen menos disponibilidad para participar en estos programas, debido a que no cuentan con el tiempo necesario, lo cual lleva a problemas en la implementación del programa. Es posible también que en los países europeos se cumplan con más facilidad las normas escolares que promueve el programa de Olweus (1993). Todo esto sugiere que puede haber contextos culturales que favorecen más que otros la implementación y el impacto de un programa. Es decir, los programas exitosos en otros contextos culturales representan una gran fuente de inspiración, pero siempre requieren evaluaciones y adaptaciones en los contextos locales donde se implementen, como las que reporta Varela.

Es claro que el contexto cultural es un factor fundamental, como lo mencionaron varios de los estudios de este compendio haciendo referencia al modelo ecológico de Bronfenbrenner (1979). Sin embargo, se requieren más investigaciones que analicen su efecto de manera sistemática. Las réplicas son útiles, pero requieren metodologías idénticas o similares para poder afirmar que los resultados difieren o no dependiendo del contexto. En cualquier caso, las similitudes entre contextos son tantas que lo encontrado en un contexto debe servir por lo menos como hipótesis preliminar para explicar lo que ocurre en otros contextos o como inspiración para considerar posibles intervenciones que luego tendrán que ser evaluadas.

\section{Implicaciones Para Políticas Públicas}

Los estudios que hacen parte de este compendio sugieren diversas ideas para ser consideradas seriamente en planes de políticas públicas. Quiero aquí resaltar tres implicaciones:

\section{Capacitación de Profesores en Ejercicio y de Profesores en Formación}

Como lo muestran varios de los estudios, los profesores, y quienes se están formando para serlo, requieren formación que les permita identificar y diferenciar los diversos tipos de agresión que ocurren en el contexto escolar y contar con herramientas prácticas para prevenir y responder adecuadamente a este tipo de situaciones. Los currículos de formación de docentes deben promover una comprensión clara y un manejo adecuado de estos temas. Todo profesor recién graduado de su licenciatura, en cualquier área, debe ser capaz de promover un clima constructivo en sus aulas, manejando constructivamente cualquier situación de agresión o indisciplina y promoviendo que los mismos estudiantes aprendan a frenar situaciones de maltrato entre compañeros. Debe, además, ser capaz de manejar pacíficamente cualquier conflicto que tenga con sus estudiantes, con colegas o con cualquier otro miembro de su comunidad educativa. Esto no solamente contribuiría a prevenir la agresión y promover la convivencia, sino que seguramente contribuiría a mejorar el desempeño académico de los estudiantes, así como la motivación, involucramiento y autoeficacia de los mismos profesores.

\section{Promoción de Programas Integrales}

Varios de los estudios muestran que la violencia escolar no puede tratarse como algo generado exclusivamente por individuos problemáticos, sino como un asunto complejo que involucra a diversos actores de la comunidad educativa y que debe tratarse con programas integrales que busquen influir en las dinámicas de grupo, en el clima de aula, en el clima escolar y en el clima familiar. Las políticas públicas sobre violencia escolar deben incluir desarrollo, adaptación y promoción de programas integrales que le lleguen a estudiantes, profesores, directivas, padres de familia y demás miembros de las comunidades educativas, incluso realizando esfuerzos adicionales con quienes más lo necesitan por estar en mayor riesgo de problemas de violencia más adelante (Chaux, 2005, en prensa). 


\section{Apoyo a Nuevas Evaluaciones}

Varela indica que se han realizado muy pocas evaluaciones rigurosas de los programas de intervención existentes. Sin estas evaluaciones no es posible saber si los programas están teniendo el efecto buscado. Es posible que se estén desperdiciando muchos recursos al no estar promoviendo los programas que más efecto podrían estar teniendo. Realizar evaluaciones rigurosas es costoso y complejo en términos técnicos, por lo cual es fundamental el apoyo del Estado. Convocatorias que garanticen el apoyo técnico de expertos (ver, por ejemplo, Social and Character Development Research Consortium, 2010) pueden tener un enorme impacto, porque garantizan que se genere capacidad en estrategias rigurosas de evaluación. Sin evaluaciones rigurosas, la legislación que se ha desarrollado y los grandes esfuerzos que se están realizando se pueden perder. En el fondo, el costo que las evaluaciones implican es bajo en comparación con el enorme beneficio que generan, tanto en la promoción de convivencia pacífica y la formación integral de ciudadanos como en la promoción de un mejor desempeño académico.

\section{Referencias}

Aguilera, M. A., Muñoz, G. \& Orozco, A. (2007). Disciplina, violencia y consumo de sustancias nocivas a la salud en escuelas primarias y secundarias de México. Ciudad de México, México: Instituto Nacional Para la Evaluación de la Educación.

Bandura, A. (1989). Human agency in social cognitive theory. American Psychologist, 44, 1175-1184. doi:10.1037//0003066x.44.9.1175

Baumrind, D. (1973). The development of instrumental competence through socialization. En A. D. Pick (Ed.), Minnesota Symposia on Child Psychology, Vol. 7 (pp. 3-46). Minneapolis, MN: University of Minnesota.

Bronfenbrenner, U. (1979). The ecology of human development: Experiments by nature and design. Cambridge, MA: Harvard University Press.

Chaux, E. (2005). El programa de prevención de Montreal: lecciones para Colombia. Revista de Estudios Sociales, 21, 11-26.

Chaux, E. (en prensa). Educación, convivencia y agresión escolar. Bogotá, Colombia: Universidad de los Andes.

Chaux, E. \& Velásquez, A. M. (2008). Violencia en los colegios de Bogotá: contraste internacional y algunas recomendaciones. Revista Colombiana de Educación, 55, 14-37.

Craig, W. M. \& Pepler, D. J. (1997). Observations of bullying and victimization in the school yard. Canadian Journal of School Psychology, 13, 41-59. doi:10.1177/082957358901300205

DeLuca, S., Pigott, T. \& Rosenbaum, J. E. (2002, Abril). Are dropout decisions related to peer threats, social isolation, and teacher disparagement across schools? A multilevel approach to social climate and dropout. Ponencia presentada en la Annual Meeting of the American Educational Research Association, New Orleans, LA, Estados Unidos.

Farrington, D. P. \& Ttofi, M. M. (2009). How to reduce school bullying. Victims \& Offenders, 4, 321-326. doi:10.1080/ 15564880903227255

Forero, R. McLellan, L., Rissel, C. \& Bauman, A. (1999). Bullying behaviour and psychosocial health among school students in New South Wales, Australia: Cross sectional survey. British Medical Journal, 319, 344-348.

Gladstone, G. L., Parker, G. B. \& Malhi, G. S. (2006). Do bullied children become anxious and depressed adults? A cross-sectional investigation of the correlates of bullying and anxious depression. The Journal of Nervous and Mental Disease, 194, 201-208. doi:10.1097/01.nmd.0000202491.99719.c3

Hawkins, D. L., Pepler, D. J. \& Craig, W. M. (2001). Naturalistic observations of peer interventions in bullying. Social Development, 10, 512-527. doi:10.1111/1467-9507.00178

Hughes, J. N. (2002). Authoritative teaching: Tipping the balance in favor of school versus peer effects. Journal of School Psychology, 40, 485-492. doi:10.1016/S0022-4405(02)00125-5

Jones, V. (2006). How do teachers learn to be effective classroom managers? En C. M. Evertson \& C. S. Weinstein (Eds.), Handbook of classroom management: Research, practice, and contemporary issues (pp. 887-907). Mahwah, NJ: Lawrence Erlbaum.

Kärnä, A., Voeten, M., Little, T. D., Poskiparta, E., Kaljonen, A. \& Salmivalli, C. (2011). A large-scale evaluation of the KiVa anti-bullying program: Grades 4-6. Child Development, 82, 311-330. doi:10.1111/j.1467-8624.2010.01557.x

Kornblit, A. L., Adaszko, D. \& Di Leo, P. F. (2008). Clima social escolar y violencia: un vínculo explicativo posible. Un estudio en escuelas medias argentinas. En C. Berger \& C. Lisboa (Eds.), Violencia escolar: estudios y posibilidades de intervención en Latinoamérica (pp. 109-138). Santiago, Chile: Editorial Universitaria.

Madriaza, P. (2008). Violencia escolar en Chile. En C. Guajardo (Ed.), Seguridad y prevención: la situación de Argentina, Chile y Uruguay durante 2007 (pp. 114-139). Santiago, Chile: Centro Internacional para la Prevención de la Criminalidad/Universidad Alberto Hurtado.

Olweus, D. (1993). Conductas de acoso y amenaza entre escolares. Madrid, España: Morata.

Rodkin, P. C., Farmer, T. W., Pearl, R. \& Van Acker, R. (2000). Heterogeneity of popular boys: Antisocial and prosocial configurations. Developmental Psychology, 36, 14-24. doi:10.1037//0012-1649.36.1.14 
Salmivalli, C., Kärnä, A. \& Poskiparta, E. (2009). From peer putdowns to peer support: A theoretical model and how it translated into a national anti-bullying program. En S. R. Jimerson, S. M. Swearer \& D. L. Espelage (Eds.), Handbook of bullying in schools: An international perspective (pp. 441-454). New York, NY: Routledge.

Salmivalli, C., Lagerspetz, K., Björkqvist, K., Österman, K. \& Kaukiainen, A. (1996). Bullying as a group process: Participant roles and their relations to social status within the group. Aggressive Behavior, 22, 1-15. doi:10.1002/ (SICI)1098-2337(1996)22:1<1::AID-AB1>3.0.CO;2-T

Social and Character Development Research Consortium (2010). Efficacy of schoolwide programs to promote social and character development and reduce problem behavior in elementary school children. Washington, DC: National Center for Education Research/Institute of Education Sciences/U.S. Department of Education.

Tremblay, R. E., Gervais, J. \& Petitclerc, A. (2008). Prevenir la violencia a través del aprendizaje en la primera infancia. Montréal, Canadá: Centre de Excellence for Early Childhood Development.

Vitaro, F., Brendgen, M. \& Tremblay, R. E. (2001). Preventive intervention: Assessing its effects on the trajectories of delinquency and testing for mediational processes. Applied Developmental Science, 5, 201-213. doi:10.1207/ S1532480XADS0504_02 